\title{
Synthesis of biodiesel from palm fatty acid distillate using sulfonated palm seed cake catalyst
}

\begin{abstract}
The use of a sulfonated soaked palm seed cake (SPSC-SO3H) derived catalyst for the production of biodiesel from palm fatty acid distillate (PFAD) (the byproduct obtained during palm oil production) has been demonstrated. The activated carbon material from the soaked palm seed cake (SPSC) was sulfonated and then used for the esterification of PFAD (containing $85 \%$ of free fatty acid (FFA), $10 \%$ of triglycerides, $3 \%$ of diglycerides, $0.3 \%$ of monoglycerides and some traces of impurities). The synthesized SPSC-SO3H catalyst was characterized using powder X-ray diffraction (XRD), Brunauer-Emmett-Teller (BET), fourier transform infrared (FTIR) spectroscopy, field emission scanning electron microscope (FESEM), NH3-temperature programmed desorption (NH3-TPD), N2 physisorption and thermogravimetric analysis (TGA). The SPSC-SO3H catalyst showed higher acid density $(12.08 \mathrm{mmol} \mathrm{g}-1)$ and surface area $(483.07 \mathrm{~m} 2 \mathrm{~g}-1)$. The optimized reaction conditions, i.e. 9:1 methanol/PFAD molar ratio; $60{ }^{\circ} \mathrm{C}$ reaction temperature; $2.5 \mathrm{wt} . \%$ of the SPSC-SO3H catalyst and $2 \mathrm{~h}$ of reaction time was employed to achieve FFA conversion (98.2\%) and FAME yield (97.8\%). The SPSC-SO3H catalyst underwent eight reaction cycles and catalytic activity was dropped by $24 \%$ during recyclability study. The SPSC-SO3H catalyst demonstrates a promising and effective application for biodiesel synthesis especially for feedstocks containing high free fatty acid content.
\end{abstract}

Keyword: Palm seed cake; Palm fatty acid distillate; Heterogeneous catalyst; Sulfonation; Reusability; Biodiesel 